\title{
Mechanisms that determine the transmembrane disposition of proteins
}

\author{
Stephen High and Bernhard Dobberstein
}

\author{
European Molecular Biology Laboratory, Heidelberg, Germany
}

\begin{abstract}
The final orientation that a protein assumes in the membrane of the endoplasmic reticulum is determined by a few types of signal sequences and their respective interactions with the membrane insertion complex. Membrane insertion occurs via a series of discrete steps, some of which are regulated by GTP- and ATP-binding proteins. Analysis of the protein components in proximity to nascent secretory and membrane proteins has revealed novel proteins in the endoplasmic reticulum that may form part of the membrane insertion complex.
\end{abstract}

Current Opinion in Cell Biology 1992, 4:581-586

\section{Introduction}

The endoplasmic reticulum (ER) is a major site of membrane biogenesis in eukaryotic cells. A key feature of this biogenesis is the vectorial insertion of proteins into the lipid bilayer of the ER. After their synthesis, membrane proteins either remain in the ER or are transported to subcellular destinations throughout the exocytic and endocytic pathways, such as the Golgi complex and lysosomes, as well as the plasma membrane.

Different proteins assume different orientations within the membrane. Integral membrane proteins that span the membrane once can expose either the amino (type I) or carboxyl (type II) terminus on the exoplasmic side of the membrane. Proteins that span the membrane several times are referred to as multiple-spanning.

The orientation of a membrane protein is defined during its insertion into the ER membrane, and is maintained, whatever the destination of the protein. The orientation that a protein assumes depends on the type of signal sequence that it bears [1]. ER-specitic signal sequences can be either cleaved or uncleaved, and are responsible for targeting proteins to the ER; they either initiate their membrane insertion, or, in the case of secreted pro. teins, their translocation across the membrane into the ER lumen [2]. All ER-targeting signal secpuences contain a stretch of apolar amino acid residues. The signals are recognized by the signal recognition particle (SRP) [3•], which targets the nascent chain-ribosome-SRP complex to the ER membrane and initiates membrane insertion $[2,4,5]$.

\section{Membrane orientation}

Single-spanning membranc protcins that have a cleavable amino-terminal signal sequence always have type I orientation (Fig. 1). A stop-transfer sequence [6] on the carboxyl-terminal side of the signal sequence aborts translocation of the nascent chain before it is complete and functions as a membrane anchor. In the absence of a stop-transfer sequence, the nascent chain is completely translocated across the membrane and enters the ER lumen (Fig. 1).

Membrane proteins with uncleaved 'signal-anchor' sequences of both type 1 and type II orientation (Fig. 1) have been identified [1]. The signal-anchor sequence mediates the ER targeting and insertion of the protein, and acts as the anchor sequence to retain the protein in the lipid bilayer. The final orientation that a signalanchor protein assumes in the membrane depends on the nature of the hydrophilic amino acid residues that flank the hydrophobic core of the signal-anchor sequence [7].

The properties of signal-anchor sequences that determine topology have been determined by analyzing mutant proteins either expressed in cells or inserted into microsomal membranes in vitro. From such studies it has been deduced that the number and type of charged amino acid residues in the regions flanking the hydrophobic core of the signal-anchor sequence determine membrane orientation $[8 \cdot, \cdot, 10]$. The more charged residues that a flanking segment contains, the more likely it is to be retained on the cytoplasmic side of the membrane.

\section{Abbreviations}

ER-endoplasmic reticulum; SRP - signal recognition particle; TRAM-translocating chain associating membrane protein. 


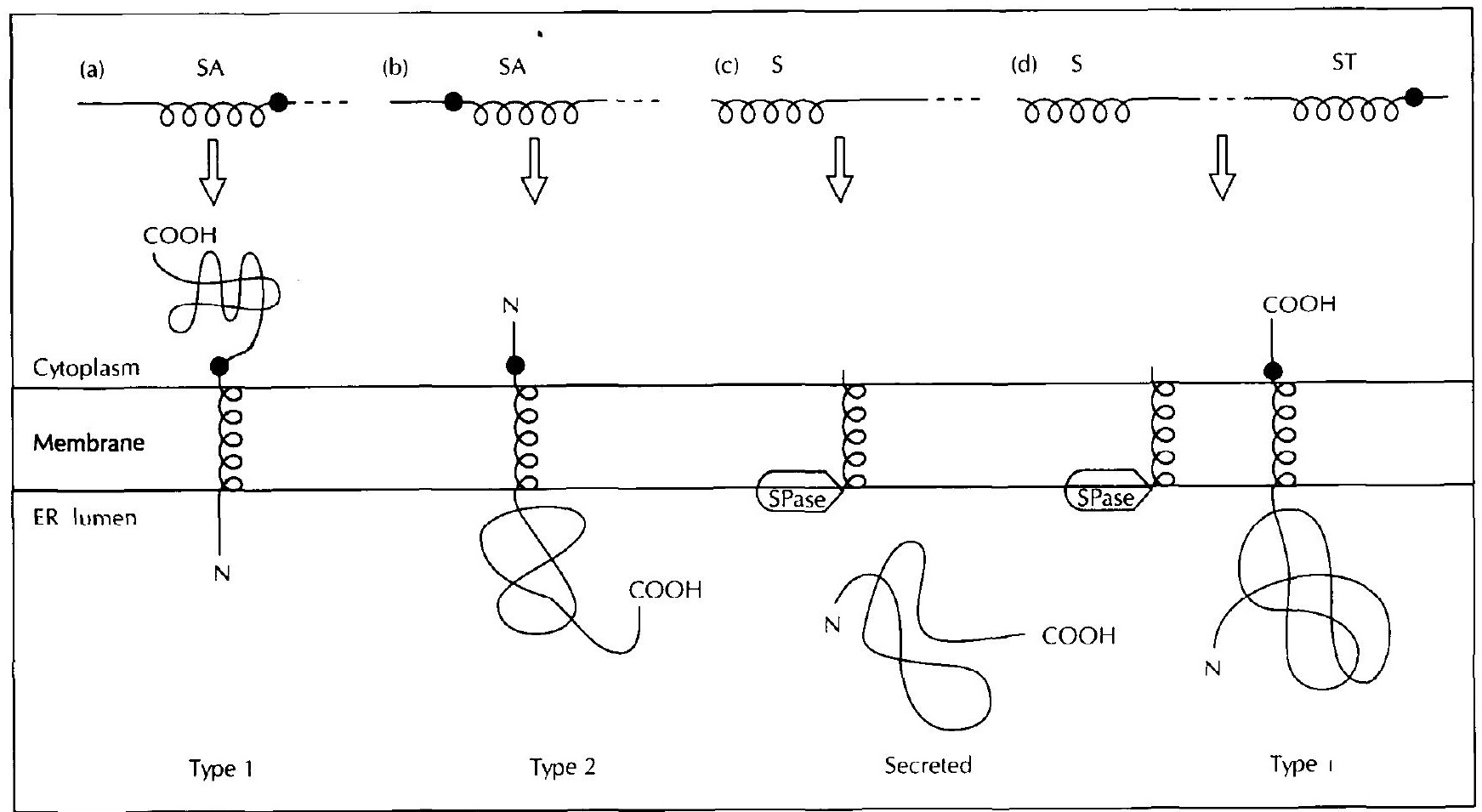

Fig. 1. Types of signal sequences. Membrane insertion in opposite orientations is mediated by (a) type I and (b) type II signal-anchor sequences (SA). Cleavable signal sequences $(\mathrm{S})$ mediate (c) the membrane translocation of secretory proteins using signal peptidase (SPase) and (d) the membrane insertion in a type I orientation, which requires a stop-transfer sequence (ST). Hydrophobic membranespanning regions are indicated by helices. O. Clusters of charged amino acid residues that often flank signal-anchor and stop-transfer sequences on the cytoplasmic side of the membrane-spanning domain. Broken lines indicate parts of the mature protein.

The correlation between the charge distribution of the regions proximal to the hydrophobic core of a signal. anchor sequence and the final orientation that the protein assumes in the membrane is strong enough to be used as the basis for predicting the membrane orientation of a protein from its amino acid sequence $[11,12]$.

In addition to the effect of charge there is also, as might be expected, a minimum length required for the hydrophobic region of a signal-anchor sequence to remain functional [10]. It has been suggested that the balance between the length of the hydrophobic seg. ment and the number of flanking charged amino acid residues determine whether a sequence functions as a signal-anchor sequence or a cleaved signal sequence [13•]. Introducing charged amino acids into a signalanchor protein does not always result in it adopting only one orientation in the membrane. Often the same protein can be found in both orientations, and the addi. tion or removal of charged residues alters the type I : type II ratio that the protein displays $[8 \cdot, 9 \bullet, 14]$.

\section{Membrane insertion as a loop}

Following proposals that the initial insertion of secretory proteins into the membrane occurs as a loop, supporting experimental evidence has been obtained [15]. The topologies observed with mutated type I and type II signal-anchor proteins $[9 \bullet, 10,13 \bullet]$ are also consistent with this model (Fig. 2). The membrane insertion of signal-anchor proteins is predicted to occur via loops formed between the hydrophobic core of the signal. anchor sequence and the flanking hydrophilic region on its amino- (type I) or carboxyl-terminal (type II) side (Fig. 2). Upon membrane insertion the final orientation is determined by which of the two regions flanking the hydrophobic core of the signal-anchor sequence is retained on the cytoplasmic side of the membrane. The difference between a secreted protein and a type II signal-anchor protein is the presence of a suitable signal peptidase cleavage site exposed on the lumenal side of the ER membrane [15].

\section{Multiple-spanning membrane proteins}

It has been proposed that muluple-spanning membrane proteins achieve their final orientation by using successive signal anchor and stop-transfer sequences [16]. There are good experimental data to support such a mechanism [17,18] and it still seems the most likely possibility [19]. Experiments with artificial chimeric proteins have shown that the hydrophilic regions between the signal-anchor and stop-transfer sequences can affect the final membrane topology of multiple-spanning mem. brane proteins [18]. This means that predictions of the orientation of multiple-spanning membrane proteins are always susceptible to errors and must be confirmed by suitable experimental approaches. 


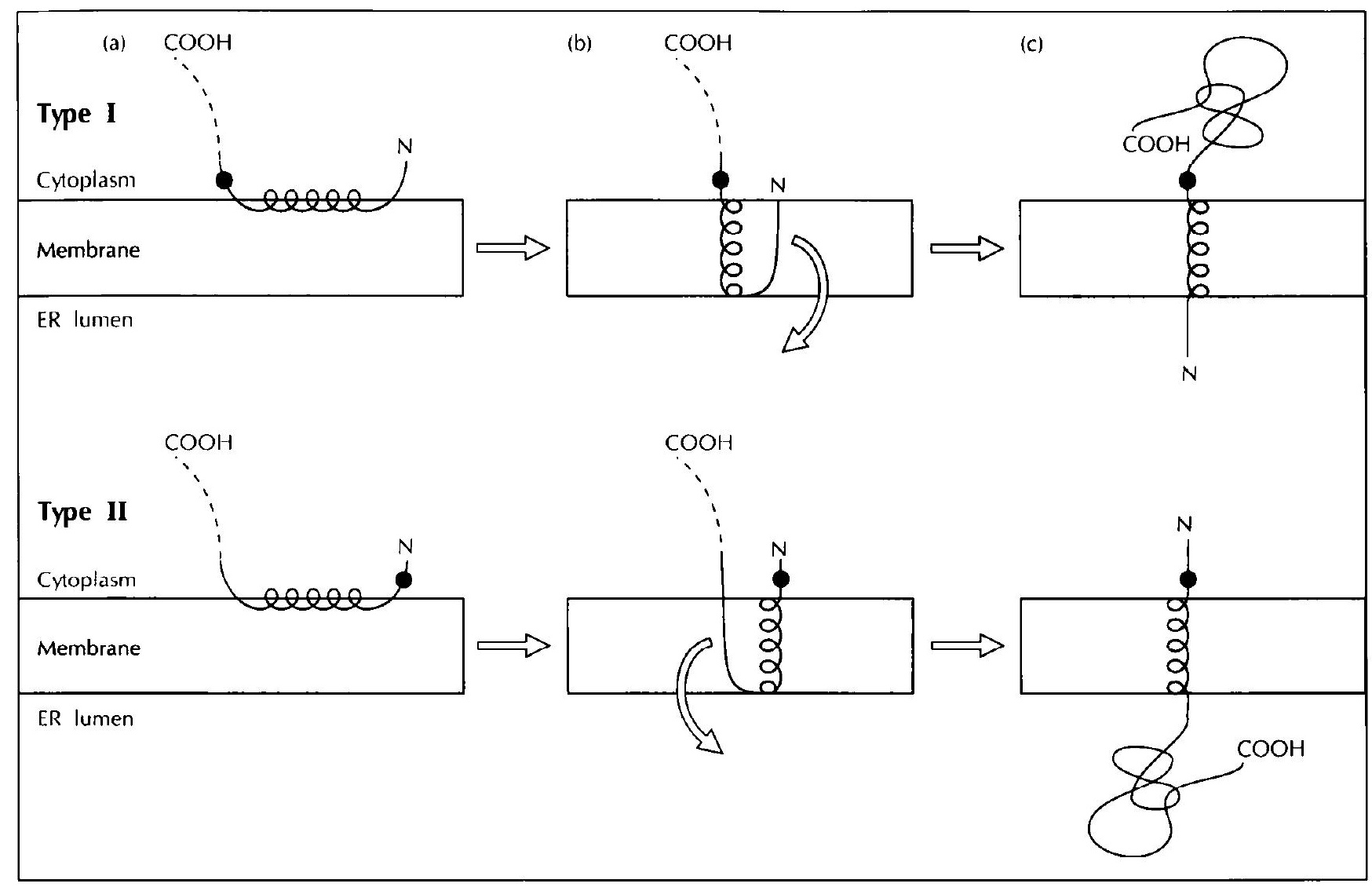

Fig. 2. Model of intermediate steps during the membrane insertion of signal-anchor proteins. The hydrophobic core of the signal-anchor sequence of a nascent protein (a) interacts with the endoplasmic reticulum (ER) membrane and (b) inserts into the membrane forming a loop with either the amino-terminal (type I) or the carboxyl-terminal (type II) flanking region of the protein. (c) The amino or carboxyl terminus of the protein is translocated into the ER lumen. O. Clusters of charged amino acid residues that often flank signal-anchor and stop-transfer sequences on the cytoplasmic side of the membrane-spanning domain.

\section{Targeting of nascent membrane proteins to the endoplasmic reticulum}

The targeting of type $\mathrm{I}$ and type II signal-anchor proteins is, like secreted proteins, mediated by SRP. These ER-targeting signals all interact with the $54 \mathrm{kD}$ subunit of SRP (SRP54) [3•]. Release of the signal sequence from the SRP54 protein requires the presence of microsomal membranes and GTP, although GTP hydrolysis is not re. quired in vitro $[20-22,23 \cdot \bullet]$.

\section{Membrane insertion}

While it is now well established that signal-anchor sequences consist of a hydrophobic core and flanking hydrophilic regions, little is known about the mechanism by which they insert into the ER membrane and attain a particular orientation across it. There is good evidence that protein components of the ER membrane are directly involved in mediating the insertion of proteins into the membrane. Thus, the translocation of secretory proteins across the ER membrane is prevented by pre-treatment of the membranes with $\mathrm{N}$-ethylmaleimide [24], which modifies the cysteine residues of proteins, or 8-azido-ATP $\left[25^{\bullet}, 26 \bullet\right]$, which is thought to block the function of ATP-binding proteins.

Nascent secretory proteins appear to be in a proteinaceous environment in the membrane since they are released from the membrane by agents that disrupt protein-protein interactions [27].

It is likely that the interaction of the nascent chain with these membrane components is also responsible for determining the final orientation of a membrane protein. The different orientations of membrane proteins could arise in at least two ways: different proteins may mediate the insertion of type I and type II signal-anchor proteins and proteins with cleaved signal sequences; or the same proteins mediate all membrane insertion and translocation events, and the details of the molecular interactions are influenced by the properties of the nascent chain.

\section{Proteins that may mediate membrane insertion}

To determine which ER proteins make up the membrane insertion machinery, cross-linking experiments have been used to define the nearest neighbours of different types of proteins during their membrane insertion. A ribosome-nascent chain-SRP complex is formed in litro and allowed to interact with the ER membrane 
to generate a stable translocation intermediate $[2,28]$. After activation of a photocross-linking reagent incorporated into the nascent chain, or cross-linking with homobifunctional reagents, the nearest neighbours can be determined. The results from a number of different laboratories $[29 \cdot \bullet-31 \cdot \bullet$ ] show that only a few ER proteins are close to the nascent chain during membrane insertion. The proteins identified by cross-linking approaches can be divided into two groups [28]: non-glycoproteins (34 to $37 \mathrm{kD}$ ) and glycoproteins $(35$ to $39 \mathrm{kD}$ ). At least one of these components, a $37 \mathrm{kD}$ non-glycoprotein (P37), is next to nascent chains with type I and type II signal. anchor sequences as well as those with cleaved signal sequences ([30••]; S High, B Dobberstein, unpublished data). The glycoproteins consist of at least two distinct proteins: the so-called signal sequence receptor (SSR $\alpha$ ) and the translocating chain associating membrane protein (TRAM; [32••].

\section{Membrane insertion complex}

The fact that at least three ER proteins (TRAM, P37 and $\operatorname{SSR} \alpha$ ) are in close proximiry in membrane inserting nascent chains suggests that a protein complex may mediate membrane insertion. In yeast, three proteins that are involved in the translocation of secreted proteins across the ER, Sec61p, Sec62p and Sec63p [33-35], form part of a complex in the ER membrane $\left[36^{\circ} \cdot\right.$. Mutations in the SEC61, SEC62 and SEC63 genes also affect the insertion of some membrane proteins $\left[37 \cdot 38^{\bullet}\right]$. suggesting that the same complex is involved in membrane protein insertion. Although no homologues of Sec61p, Sec62p or Sec63p have yet been identified in mammalian $E R$, it is tempting to speculate that the non-glycoproteins identified by cross-linking are the mammalian counterparts of at least some of these proteins [4].

\section{Mechanisms}

While it remains to be established that the proteins identi fied by cross-linking are involved in the insertion of mem brane proteins into the ER, the results described above suggest that a common machinery mediates membrane insertion. How could such a membrane insertion complex facilitate insertion in a type I or type II orientation? A charged region of the membrane insertion complex, present at the cytoplasmic face, could retain a charged region of the nascent chain adjacent to the hydrophobic core of the signal-anchor sequence (Fig. 2). The other end of the nascent chain would then be translo- same machinery is responsible for the membrane insertion of type I and type II signal-anchor proteins, and that the way in which a nascent chain interacts with components of this machinery determines its final orientation in the membrane.

An interaction between a charged region of the membrane insertion complex and the cluster of charged residues that normally follows the apolar region of a stop-transfer sequence would also account for the ability of a stop-transfer sequence to integrate into the lipid bilaver in a stable fashion [19]. Thus, one translocation site would mediate the insertion of all types of membrane proteins in a manner determined by the properties of the nascent chain itself.

\section{Conclusion}

The principal features of signal-anchor sequences, cleaved signal sequences and stop-transfer sequences have been elucidated recently. In each case it has been found that a hydrophobic core region combined with flanking hydrophilic sequences is important for function. How these different sequences function in the process of membrane insertion is not known. An attractive possi. bility is that their interaction with components of the membrane insertion complex determines the final ori entation that a protein assumes in the membrane. With the prospect that the components of this complex will be identified in the near future we can look forvard to understanding the molecular interactions that determine membrane protein orientation in the ER.

\section{Acknowledgements}

We thank foen luirink and klaus Fiedler for their careful reading of the manuscript. This work was supported by a grane from the Deutsche Forschungsgeme imschaft (Do 199 5.2, 5713 352).

\section{References and recommended reading}

Papers of particular interest, published within the annual period of to view, have been highlighted as:

- of special interest

- of outstanding interest

1. lon Hillas: G: Transcending the Imoenetrable: How Pro. lective retention of one of the two hydrophilic regions flanking the hydrophobic core of the signal-anchor sequence may determine the membrane orientation.

The observation that one signal-anchor protein can as sume two orientations in the membrane $[8 \bullet, 9 \bullet, 14]$ sug. gests that kinetic or thermodynamic competition occurs between the translocation of the amino and carboxyl termini. This is consistent with the suggestion that the
Acla 1988, 974:307-333.

2. HIGH S, DOBBlESTEIN B: Membrane Protein Insertion into the Endoplasmic Reticulum - Signals. Machinery and Mechanisms. In Hembrame Biogenesis and Protein Target. ing. Edited by Neupert W, Ijll R. Ansterdam: Elsetier, 1992, in press.

3. I.TCKE H, HIGH S, ROMISCH K, ASHFORJ AJ, DOBBH:RSTIN B:

- The Methionine-rich Domain of the $54 \mathrm{kDa}$ Subunit of Signal Recognition Particle is Sufficient for the Interaction with Signal Sequences. FMBO / 1992, $11: 1543-1551$. 
The methionine rich carboxyl-terminal domain of the $51 \mathrm{kD}$ subunit of $S R P$ is both necessary and sufficient for recognizing ER-specific signal secpuences. This shows that the amino kerminal GTP.binding donain of SRP54 is mot rexpuired for signtal seceuence bindling and must play some other role, for example, in the targeting process.

4. Gilmokl R: The Protein Translocation Apparatus of the Rough Endoplasmic Reticulum. Its Associated Proteins, and the Mechanism of Translocation. Curr Opin Cell Biol 1991. 3:580-584

5. RAPOPOKT TA: Protein Transport Across the ER Membrane Trends Biride'm Sit 1990, 15:355-358.

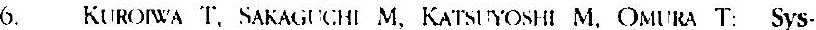
tematic Analysis of Stop-transfer Sequence for Microsomal Membrane. I Biol Clem 1991, 266:9251-9255.

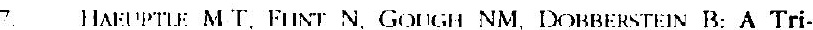
partite Structure of the Signals that Determine Protein Inscrtion into the Endoplasmic Reticulum Membrane. I Cell Biol 19\%), 108:1227 1236.

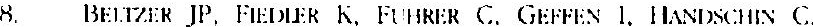
Wlistils IIP, Spliss M: Charged Residues are Major Determinants of the Transmembrane Orientation of a Signal-anchor Domain. I Biol Chem 1991, 266:973-978

The intresluction of tharged amino atid residues adjacent $t$ the hrdophobic core of the signal anchor sequence can reverse the orien. tation of the asiatoglycoprotein recepter 111 subunit, which is normally a type II membrane protein.

9. PAkh' GD, LAMls RA: Topology of Eukaryotic Type II - Membrane Proteins: Importance of N-terminal Positively Charged Residues Flanking the Hydrophobic Domain. Cell 1901, 64: $7-78$ ?

$A$ positive charge in a protein segment next to the hydrophobic core of a signal anchor sequence acts to retain that segment on the cotoplasmic side of the membrine.

10. Sato T, Saksinchi M. Mihaka $k$, Omlira T: The amino-Ter minal Structures that Determine Topological Orientation of Cytochrome P-450 in Microsomal Membrane. EVIBO / 1990, $9: 2391-239^{-}$

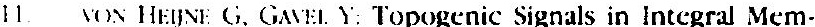
brane Protcins. Lar I Bickhem 1988, 174:671-678.

12. HAKTMAN E. RAPOPORT TA. IA) ISH HF: Predicting the Orientation of Eukaryotic Membrane-spanning Proteins. Prex Natl fiad Sid (SA 1989, 86:5786-570).

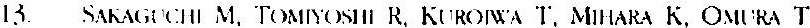
- Functions of Signal and Signal-anchor Sequences are Determined by the Balance between the Hydrophobic Segment and the N-terminal Charge. Pro Nall Acad Sici ISA 1992. 89: $1(0-19)$

A detailed analysin of the effect of adjacent charged amino acid residues and length of the hodrophobic region upon the function of a signal anchor secfuence Both properties are shown on influence whesher the I:R specific sequence functions as a type I signal anchor sequence or as a cleaved signal seculence.

14. PAKKS GD. HILI JI). LANHB RA: Transposition of Domains be tween the M2 and HN Viral Membrane Proteins Results in Polypeptides which Can Adopt more than One Membrane Orientation. / Cell Biol 1989). 109:2023-2032.

15. SHAW AF. RotTER PIM. ROSE JK: Evidence for the Loop Model of Signal-sequence Insertion into the Endoplasmic Reticulum. Prox Nall Acad Sci l'SA 1988, 85:7592-7596

16. Bı.,oв:1. G: Intracellular Protein Topogenesis. Pro Natl Acad Sci 1 S.4 1980, 77:1496-1500.

17. WESSELS HP, SPIESS M: Insertion of a Multispanning Membrane Protein Occurs Sequentially and Requires only One Signal Sequence. Cell 1988, 55:61-70

18. Lapy J, FuNT N, HafLIPTIS M.T. DOBBERSTEIN B: Struc. tural Requirements for Membrane Assembly of Proteins
Spanning the Membrane Several Times. J Cell Biol 1989. 109:2013-2022.

19. SINGr:R SJ: The Structure and Insertion of Integral Proteins into Membranes. Annu Ret Cell Biol 1990, 6:247-296.

20. WIISON C, CONNOLIY T, MORRISON T, GILMORE R: Integration of Membrane Proteins into the Endoplasmic Reticulum Requires GTP. I Cell Biol 1988, 107:69-77.

21. IIIGH S. FUNT N, DORBERSTEIN B: Requirements for the Membrane Insertion of Signal-anchor Type Proteins. / Cell Biol $1991,113: 25-34$

22. Connoly T, GLLOKE: R: The Signal-recognition Particle Receptor Mediates the GTP-dependent Displacement of SRP from the Signal Sequence of the Nascent Polypeptide. Cell 1989. $57: 599-610$

23. CONNOINY T, RAPli!ko PI. GILMORE R: Requirement of GTP -. Hydrolysis for Dissociation of the Signal Recognition Particle from Its Receptor. Science 1991, 252:1171-1173.

The hydrolysis of GTP is required to allow the dissociation of SRP from its receptor the docking protein (SRP receptor). While non.hydro. lysable analogues of GTP are sufficient to mediate membrane insertion in iviro, these results suggest that GTP hydrolysis is necessary to drive protein translocation in tito.

24. NICCHITA $\mathrm{CV}$, BI.OBHEL. G: Nascent Secretory Chain Binding and Translocation are Distinct Processes: Differentiation by Chemical Alkylation. I Cell Biol 1989, 108:789-795.

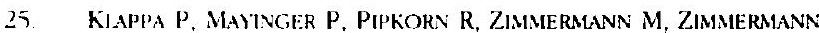
- R: A Microsomal Protein is Involved in ATP-dependent Transport of Presecretory Proteins into Mammalian Microsomes. LUHBO / 1991, 10:2795-2803.

Treatment of microsomal menbranes with azido-ATP inhibits the translixation of secretory proteins into the lumen. This is true both for proteins that show SRP-dependent transport and for small proteins that are transported independently of SRP. The results suggest an ATP.binding protein may be required for translocation.

20. ZINMI:RMAN: DL. WATER P: An ATP.binding Ptotein is Re- quired for Protein Translocation Across the Endoplasmic Reticulum. Cell Regul 1991, 2:851-859.

Treatment of nicrosomal membranes with azido-ATP inhibits the SRP. mediated transkocation of secretory proteins. Approximately 20 proteins are modified with azido-ATP, of which two are identified: the $35 \mathrm{kD}$ sig. nal sequence receptor $\alpha$-subunit and a $180 \mathrm{kD}$ protein previously shown (1) possess ribosome binding accisity:

27. GllstoRl: R. BLOBEL G: Translocation of Secretory Proteins Across the Microsomal Membrane Occurs Through an Environment Accessible to Aqueous Perturbants. Cell 1985. $42: 497-505$.

28. HiGts S: Membrane Protein Insertion into the Endoplasmic Reticulum - Another Channel Tunnel? Bioessays 1992, in press.

29. THRIFT RN, ANDRFWS DW, WALTER P, JOHNSON AE: A Nascent - Membrane Protein is Located Adjacent to ER Membrane Proteins throughout Its Integration and Translation. $J$ Cell Biol 1991, 112:809-821

A $39 \mathrm{~kJ}$ ) glycoprotein (mp39-like glycoprotein), as well as unidentified non glycoproteins, are adjacent to a membrane protein with a cleaved signal sequence and to a npe Il signal-anchor protein during mem. brane insertion. These results provide evidence that the association of nascent chains with the membrane insertion complex is transient, and that the exit of the nascent chain is coupled to the completion of protein synthesis.

30. High S, GORJCH D, WIEDMANN M, RAPOPORT TA, DOBbERSTEIN -. B: The Identification of Proteins in the Proximity of SignalAnchor Sequences during Their Targeting to and Insertion into the Membranc of the ER. / Cell Biol 1991, 113:35-44. A novel $37 \mathrm{kD}$ non-glycosylated protein (P37) is identitied as a prob. able component of the membrane insertion complex. P37 interacts with the nascent chain of a type 1 signal-anchor protein at the cyto. plasmic side of the membrane. The resulss suggest that the membrane insertion complex may be composed of heterologous protein subunits. 
31. KELLARIS KV, BOWEN S, GILMORE R: ER Transtocation Inter-. mediates are Adjacent to a Nonglycosylated 34.kD Integral Membrane Protein. I Cell Biol 1991, 114:21-33.

A novel $34 \mathrm{kD}$ non-glycosylated protein (imp34) is found adjacent to proteins that bear a cleavable signal sequence. This component is probably the same as the P37 component, and, if so, this protein is expected to be a general component of the ER translocation complex and not restricted to interacting with some signal-anchor type mem. brane proteins.

32. GORUCH D, HARTMANN E, PREHN S, RAPOPORT TA: A protein of the endoplasmic reticulum involved early in polypeptide transiocation. Nature 1992, 357:47-52.

A mammalian cell free protein synthesizing system and reconstitution into proteoliposomes was used to identify and characterize TRAM. This protein is the first multispanning membrane glycoprotein that is shown to stimulate or to be involved in membrane translocation of secretor: proteins.

33. Deshates RJ, SCHEkMuN R: A Yeast Mutant Defective at an Early Stage in Import of Secretory Protein Precursors into the Endoplasmic Reticulum. / Cell Biol 1987. 105:633-645.

34. Rothblatt JA, Deshaies RJ, Sanders Sl, Dalin G, SCHEkMaN R: Multiple Genes are Required for Proper Insertion of Secretory Proteins into the Endoplasmic Reticulum in Yeast. I Cell Biol 1989, 109:2641-2652

35. DESHAIES RJ, SCHEKMAN R: SEC62 Encodes a Putative Membrane Protein Required for Protein Translocation into the Yeast Endoplasmic Reticulum. I Cell Biol 1989. 109:2653-2664.

36. DESHAIES RJ, SANDERS SL, FELDHEIM DA, SCHEKMAN R: ASsembly -. of Yeast SEC Proteins Involved in Translocation into the Endoplasmic Reticulum into a Membrane-bound Complex. Nature 1991, 349:806-808.

Sec61p, Sec62p and Sec63p are shown to be part of a multisubunit protein complex present in the ER, believed to be part of the trans- location complex. The results confirm genetic evidence for an interaction between the products of these SEC genes, and demonstrate that the translocation complex of the ER membrane is a complicated multisubunit structure.

37. STIRUNG CJ, ROTHBLATt J, HOSOHIICHI M, DEshaifs R, SCHEkMLAN

- R: Protein Translocation Mutants Defective in the Insertion of Integral Membrane Proteins into the Endoplasmic Reticulum. . Iol Biol Cell 1992, 3:129-142

The Sec61p, Sec62p, and Sec63p gene products, previously shown to be necessany for the translocation of secreted proteins, are shown to be required for the insertion of some membrane proteins. This sug. gests that a common machinery is at least in part responsible for the translocation of secreted proteins across the ER membrane and the in. sertion of membrane proteins into it. The SEC61 gene is shown to be essential for cell growth and encoding a hudrophobic ER membrane protein of $38 \mathrm{kD}$ (apparent molecular weight). The protein bears some resemblance to the Esterichia coli Sect protein. which forms a part of the putative secteton protein translocation complex of the bacterial inner nembrane.

38. Greex N, Favi H, Watthe P: Mutants in Three Novel Com- plementation Groups Inhibit Membrane Protein Insertion into and Soluble Protein Translocation across the Endoplasmic Reticulum Membrane of Saccharomyces cerevisiae. I Cell Biol 1992, 116:59?-604.

Mutants of the SECGI and SECG3 genes affect the insertion of mem. brane proteins, consistent with a common machinen for the complete translocation of secreted proteins across the ER and the insertion of nembrane proteins into it. In addition, three new mutants that inhihit membrane protein insertion were identified; SEC 70, SEC 71 and SEC ? It is not yet known whether these mutations affect the targeting or the membranc insertion process.

S High and B Dobberstein, European Molecular Biolog: Laboratong: Meyerhofstrasse 1. 6900 Heidelherg. Germany: 